\title{
New Constraints on Preferred Frame Effects from Binary Pulsars
}

\author{
Lijing Shao ${ }^{1}$, Norbert Wex $^{2}$, Michael Kramer ${ }^{3}$ \\ ${ }^{1}$ Max-Planck-Institut für Radioastronomie, Auf dem Hügel 69, 53121 Bonn, Germany \\ School of Physics, Peking University, Beijing 100871, China \\ email: 1shao@pku.edu.cn \\ ${ }^{2}$ Max-Planck-Institut für Radioastronomie, Auf dem Hügel 69, 53121 Bonn, Germany \\ email: wex@mpifr-bonn.mpg.de \\ ${ }^{3}$ Max-Planck-Institut für Radioastronomie, Auf dem Hügel 69, 53121 Bonn, Germany \\ Jodrell Bank Centre for Astrophysics, School of Physics and Astronomy, \\ The University of Manchester, M13 9PL, UK \\ email: mkramer@mpifr-bonn.mpg.de
}

\begin{abstract}
Preferred frame effects (PFEs) are predicted by a number of alternative gravity theories which include vector or additional tensor fields, besides the canonical metric tensor. In the framework of parametrized post-Newtonian (PPN) formalism, we investigate PFEs in the orbital dynamics of binary pulsars, characterized by the two strong-field PPN parameters, $\hat{\alpha}_{1}$ and $\hat{\alpha}_{2}$. In the limit of a small orbital eccentricity, $\hat{\alpha}_{1}$ and $\hat{\alpha}_{2}$ contributions decouple. By utilizing recent radio timing results and optical observations of PSRs J1012+5307 and J1738+0333, we obtained the best limits of $\hat{\alpha}_{1}$ and $\hat{\alpha}_{2}$ in the strong-field regime. The constraint on $\hat{\alpha}_{1}$ also surpasses its counterpart in the weak-field regime.
\end{abstract}

Keywords. Gravitation, pulsars: general, pulsars: individual (J1012+5307, J1738+0333)

\section{Introduction}

Pulsars are extremely stable electromagnetic emitters, and along with their extreme physical properties and surrounding environments, they provide useful astrophysical laboratories to study fundamental physics (Lorimer \& Kramer 2005). Radio timing of binary pulsars maps out the binary orbital dynamics through recording the time-of-arrivals of the pulsar signals at the telescope. For millisecond pulsars this can be done with high precision, providing a powerful tool to probe gravity (see e.g., Stairs 2003 and Kramer et al. 2006). In this work, we summarize new results on testing the local Lorentz invariance (LLI) of gravity from binary pulsars obtained by Shao \& Wex (2012).

\section{New Limits on Preferred Frame Effects}

Non-gravitational LLI is an important ingredient of the Einstein equivalence principle (EEP) (Will 1993, 2006). But even metric gravity, which fulfills the EEP exactly, could still exhibit a violation of LLI in the gravitational sector (Will \& Nordtvedt 1972, Nordtvedt \& Will 1972, Damour \& Esposito-Farèse 1992, Will 1993). Such a violation of LLI induces preferred frame effects (PFEs) in the orbital dynamics of a binary system that moves with respect to the preferred frame. In the parametrized post-Newtonian (PPN) formalism, PFEs of a semi-conservative gravity theory are described by two parameters, $\hat{\alpha}_{1}$ and $\hat{\alpha}_{2} \cdot \dagger$

$\dagger$ To distinguish from their weak-field counterparts $\left(\alpha_{1}\right.$ and $\left.\alpha_{2}\right)$, here "hat" indicates possible modifications by strong-field effects. 
The orbital dynamics of binary pulsars with non-vanishing $\hat{\alpha}_{1}$ and $\hat{\alpha}_{2}$ are obtained from a generic semi-conservative Lagrangian (Damour \& Esposito-Farèse 1992). It is found that in the limit of small orbital eccentricity, PFEs induced by $\hat{\alpha}_{1}$ and $\hat{\alpha}_{2}$ decouple, and lead to separable effects in the timing observations. Hence they can be tested independently using observations of only one binary pulsar (Shao \& Wex 2012).

- A non-zero $\hat{\alpha}_{1}$ induces a polarization of the eccentricity vector towards a direction in the orbital plane perpendicular to the velocity of the binary system with respect to the preferred frame, $\mathbf{w} . \dagger$ The observed eccentricity vector $\mathbf{e}(t)$ is a vectorial superposition of a "relativistically rotating" eccentricity $\mathbf{e}_{R}(t)$ (of constant length) and a "fixed eccentricity" $\mathbf{e}_{F} \propto \hat{\alpha}_{1}: \mathbf{e}(t)=\mathbf{e}_{R}(t)+\mathbf{e}_{F}$ (Damour \& Esposito-Farèse 1992). The effect is graphically illustrated in the left panel of Fig. 1. Previous methods use the smallness of the observed eccentricity, combined with probabilistic considerations concerning the unknown angle $\theta$ (the angle between $\mathbf{e}_{R}$ and $\mathbf{e}_{F}$ ) to constrain $\hat{\alpha}_{1}$ (Damour \& Esposito-Farèse 1992, Wex 2000). The method developed in Shao \& Wex (2012) is an extension of the method by Damour \& Esposito-Farèse (1992) that does not require any probabilistic considerations concerning $\theta$. It is applicable to binary pulsars of short orbital period that have been observed for a long enough time, during which the periastron has advanced significantly. Even if the advance of periastron is not resolved in the timing observation, the constraints on the (observed) eccentricity vector can be converted into a limit on $\hat{\alpha}_{1}$. From the 10 years of timing and the optical observations of PSR J1738+0333 (Antoniadis et al. 2012, Freire et al. 2012) one obtains the most constraining limit,

$$
\hat{\alpha}_{1}=-0.4_{-3.1}^{+3.7} \times 10^{-5} \quad(95 \% \text { C.L. }),
$$

which is significantly better than the current best limits from both weak field (Müller et al. 2008) and strong field (Wex 2000).

- A non-vanishing $\hat{\alpha}_{2}$ induces a precession of the orbital angular momentum around the direction of $\mathbf{w}$ (see the right panel of Fig. 1), which changes the observed orbital inclination. Using the long-term timing results on PSRs J1012+5307 (Lazaridis et al. 2009) and J1738+0333 (Antoniadis et al. 2012, Freire et al. 2012), Shao \& Wex (2012) find an upper limit

$$
\left|\hat{\alpha}_{2}\right|<1.8 \times 10^{-4} \quad(95 \% \text { C.L. })
$$
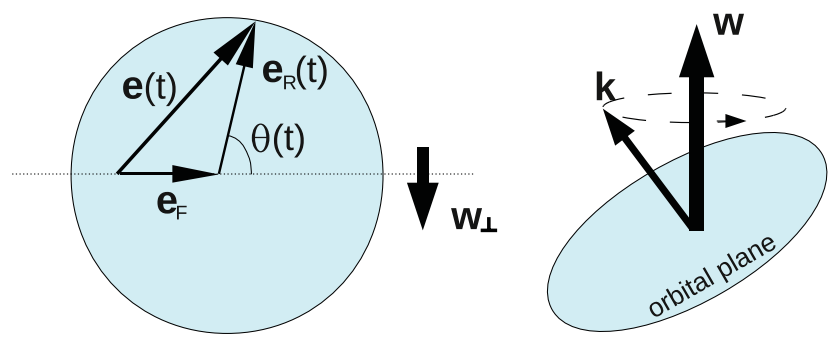

Figure 1. Illustration of preferred frame effects in the orbital dynamics of small-eccentricity binary pulsars; see Shao \& Wex (2012) for details. Left: $\hat{\alpha}_{1}$ tends to polarize the orbital eccentricity vector $\mathbf{e}(t)$ towards the direction perpendicular to $\mathbf{w}_{\perp}$ (Damour \& Esposito-Farèse 1992); right: $\hat{\alpha}_{2}$ induces a precession of the orbital angular momentum around the direction of $\mathbf{w}$.

$\dagger$ Here we choose the isotropic cosmic microwave background as the preferred frame; nevertheless, see Shao \& Wex (2012) for constraints on other preferred frames. 
which is better than the current best limit for strongly self-gravitating bodies (Wex \& Kramer 2007) by more than three orders of magnitude, but still considerably weaker than the weak-field limit of $\alpha_{2}$ by Nordtvedt (1987).

\section{Summary}

We summarize results presented in Shao \& Wex (2012) that proposed new tests of LLI. These yield improved constraints on PFEs from binary pulsar experiments. Specifically, limits on parameters $\hat{\alpha}_{1}$ and $\hat{\alpha}_{2}$ are obtained from long-term timing of two binary pulsars with short orbital period (see Eqs. (2.1) and (2.2)). Our extended $\hat{\alpha}_{1}$ test no longer requires probabilistic considerations related to unknown angles. The proposed tests have the advantage that they continuously improve with time, and will benefit greatly from the next generation of radio telescopes, like FAST (Nan et al. 2011) and SKA (Smits et al. 2009).

\section{Acknowledgment}

Lijing Shao is supported by China Scholarship Council (CSC).

\section{References}

Antoniadis, J., van Kerkwijk, M. H., Koester, D., Freire, P. C. C., Wex, N., Tauris, T. M., Kramer, M., \& Bassa, C. G. 2012, MNRAS, 423, 3316

Damour, T. \& Esposito-Farèse, G. 1992, Phys. Rev. D, 46, 4128

Freire, P. C. C., Wex, N., Esposito-Farèse, G., Verbiest, J. P. W., Bailes, M., Jacoby, B. A., Kramer, M., Stairs, I. H., Antoniadis, J., \& Janssen., G. H. 2012, MNRAS, 423, 3328

Kramer, M., Stairs, I. H., Manchester, R. N., McLaughlin, M. A., Lyne, A. G., Ferdman, R. D., Burgay, M., Lorimer, D. R., Possenti, A., D’Amico, N., Sarkissian, J. M., Hobbs, G. B., Reynolds, J. E., Freire, P. C. C., \& Camilo, F. 2006, Science, 314, 97

Lazaridis, K., Wex, N., Jessner, A., Kramer, M., Stappers, B. W., Janssen, G. H., Desvignes, G., Purver, M. B., Cognard, I., Theureau, G., Lyne, A. G., Jordan, C. A., \& Zensus, J. A. 2009, MNRAS, 400, 805

Lorimer, D. R. \& Kramer, M. 2005, Handbook of Pulsar Astronomy (Cambridge University Press)

Müller, J., Williams, J. G., \& Turyshev, S. G. 2008, in: H. Dittus, C. Lammerzahl, \& S. G. Turyshev (eds.), Lasers, Clocks and Drag-Free Control: Exploration of Relativistic Gravity in Space 349 (Astrophysics and Space Science Library: Springer), p. 457

Nan, R., Li, D., Jin, C., Wang, Q., Zhu, L., Zhu, W., Zhang, H., Yue, Y., \& Qian, L. 2011, International Journal of Modern Physics D, 20, 989

Nordtvedt, K. 1987, ApJ, 320, 871

Nordtvedt, K. \& Will, C. M. 1972, ApJ, 177, 775

Shao, L. \& Wex, N. 2012, Class. Quantum Grav., accepted [arXiv:1209.4503]

Smits, R., Kramer, M., Stappers, B., Lorimer, D. R., Cordes, J., \& Faulkner, A. 2009, A\&A, 493,1161

Stairs, I. H. 2003, "Testing General Relativity with Pulsar Timing”, Living Rev. Relativity 6, 5. URL (cited on 2012-09-11): http://www.livingreviews.org/lrr-2003-5

Wex, N. 2000, in: M. Kramer, N. Wex, \& R. Wielebinski (eds.), IAU Colloq. 177: Pulsar Astronomy - 2000 and Beyond 202 (Astronomical Society of the Pacific Conference Series: San Francisco), p. 113

Wex, N. \& Kramer, M. 2007, MNRAS, 380, 455

Will, C. M. 1993, Theory and Experiment in Gravitational Physics (Cambridge University Press)

Will, C. M. 2006, "The Confrontation between General Relativity and Experiment", Living Rev. Relativity 9, 3. URL (cited on 2012-09-06): http://www.livingreviews.org/lrr-2006-3

Will, C. M. \& Nordtvedt, K. 1972, ApJ, 177, 757 